Author's copy of Book review as accepted for publication - authored by Chronoula Voutsina

Book Review

\title{
A Practical Introduction to In-Depth Interviewing
}

by Alan Morris

London, Sage, 143 pp., ISBN 978-1-4462-8763-7 (pbk)

This book aims at providing a practical guide to the processes involved in planning and conducting in-depth semi-structured interviews. As the author indicates in the Preface, the book is written for social science researchers with limited experience or confidence in using interviews as a method of data collection. Structured in 8 chapters, and drawing on actual qualitative studies, the book presents an accessible account of the main stages and considerations involved in designing and conducting interviews and addresses the author's aim to facilitate the development of skills that are required for the successful use of the method.

Chapter 1 presents a brief historical account of the use of interviews and describes different interview approaches in relation to their scope, aim and structure before discussing characteristics of in-depth, semi-structured interviews that are the focus of this book. In the section titled: 'What is an in-depth interview' the reader may query the author's approach to associate the attribute 'indepth' with semi-structured interviews exclusively as it is not clear why this attribute would not be equally associated with narrative, life history or other approaches to interviewing. Indeed, one might argue that the aim of using any type of qualitative interviews in social sciences, generally, is nothing but the collection of in-depth data.

In Chapter 2, the author discusses key principles of ethical conduct at all phases of research, including issues that may arise during the interview and the writing up stage. The chapter also includes a helpful guide into ethical approval procedures. The inclusion of a chapter on Ethics early in the order of chapters is a positive feature of the book and highlights for the novice researcher the 
Author's copy of Book review as accepted for publication - authored by Chronoula Voutsina

importance of ethical conduct as a concept that goes beyond the processes of institutional ethical approval. The need to design, conduct and report on interviews with sensitivity, honesty and respect to the research participants and their social and cultural contexts is not restricted to this chapter only. Rather, it is a theme that is revisited at different sections throughout the book; for example, when discussing issues related to recruitment in Chapter 4 and accessing difficult to reach groups in Chapter 7.

In Chapter 3, the author provides helpful advice on how to design an interview guide and write interview questions of different types that are relevant to the research aims. Chapter 4 on selecting and accessing research participants, and Chapter 5 on how to prepare for the interview, offer practical guidance on how the interviewer can establish a rapport of trust and respect with the interviewee; one of the key contributing factors to a successful interview.

Chapter 6 illustrates with useful examples principles and techniques of effective interviewing such as the logical ordering of questions and probing strategies. Advice on how to deal with unexpected events and discussion on factors that may present challenges in accessing and interviewing particular groups of participants is offered in Chapter 7. The section on 'Interviewing across difference' addresses issues related to the need for the interviewer to be "sensitive to difference and power differentials" (p. 107). Given that the author acknowledges that 'difference' can be an enabling rather than constraining factor at different circumstances, the reader may query here the choice to address issues of 'difference' within a chapter that is titled 'Dealing with difficulties and the unexpected', rather than as part of the previous chapter that deals with good, skilful interviewing conduct in all cases. The discussion at certain points would benefit from added nuance and elaboration. For example, it would have been good if the underlying common principles of community-based participatory research (CBPR) for interviewing indigenous communities and participatory research for interviewing members of the GLBT community had been unpicked. 
Author's copy of Book review as accepted for publication - authored by Chronoula Voutsina

Chapter 8 addresses the processes of transcribing, analysing and writing up the interviews. Basic principles of thematic analysis and coding are outlined. A drawback might be that there is limited space in the book (or indeed it is beyond its scope) to describe different approaches to analysis or address related methodological issues, such as the implications that the positioning of interview data within different adopted conceptual frameworks might have to the analysis. The need to conduct the interview and approach the data 'reflexively' is mentioned at different points in the book, however, novice researchers, who are the primary audience, will need to resort to other, methodological, literature to develop an understanding of this concept.

One of the strongest features of the book is that, across all chapters, it includes well selected examples from the author's own extensive research aiming to facilitate the reader's understanding of proposed practices such as designing an interview guide in Chapter 3 and record keeping in Chapter 8. Extracts from actual interviews are used very effectively in Chapter 6 to illustrate practices that may affect the quality of interview data, such as the use of effective probing techniques. At the end of each chapter, the reader will find a suggestion for a well-focused exercise that aims at facilitating the application of relevant skills.

The book succeeds in its aim to provide a practical introduction to in-depth interviewing. While the reader will need to resort to other sources for an understanding of methodological issues associated with the use of interviews, the book offers a useful insight into key considerations that researchers will need to engage with in order to conduct interviews in an ethical, methodical and valid way. The book constitutes a useful reference text for introductory modules on qualitative methods and it is to be recommended for aspiring qualitative researchers in the social sciences. 